\title{
Overcoming of Areas Covered by Snow with the Perspective Materials
}

\author{
Klara CIBULOVA ${ }^{1}$, Martin PRIESNER ${ }^{2}$, Ota ROLENEC ${ }^{3}$ \\ 1, 2, 3 Department of Engineer Technology, Faculty of Military Technology, University of Defence in Brno, Address: \\ Kounicova 65, 66210, Brno, Czech Republic
}

E-mails: ${ }^{1}$ klara.cibulova@unob.cz; ${ }^{2}$ martin.priesner@unob.cz; ${ }^{3}$ ota.rolenec2@unob.cz

\begin{abstract}
The support of mobility is one of the main tasks of engineer units. The mobility consists of different parts - mobility of low-endurable terrain, watercourses, forests etc. In this paper the authors focused only to one area - trafficability of areas covered by snow. The snowy terrain covered most of areas, not only in the Czech Republic, during the winter season. But the mobility must be ensured even in such conditions. In some cases, the vehicles mire in the snow layer and they have to be rescued. In the others, the convoy of vehicles stop before the untrafficable place and they have to overcome it. It is not always possible to bypass the place and the units have to negotiate this area. But the removal of snow is very time and human consuming. So it was decided to undergo testing materials suitable for these purposes. The aim of this work is to evaluate the using of perspective materials for self-rescuing works in snow as well as for the negotiation of untrafficable terrain due to the layer of snow. And all this makes it possible to improve the ability of negotiation obstacles such as snowy terrain and thus better mobility of the troops even in such conditions. $[1,2,3]$
\end{abstract}

KEY WORDS: trafficability, negotiation of snow, perspective materials

\section{Introduction}

The mobility is one of the basic features of the troops. The main mobility assumption is trafficability. Trafficability is the ability of the given area to support vehicle through it. The trafficability consist of different types - towns, woods, forests, waters, etc. The authors are dealing with these types one after another. In this paper they evaluate the possible ways of overcoming areas covered by snow. During winter seasons the troops often have to overcome the areas with snow. It depends a lot on the high of the snow layer, the weather condition - if it is frozen or melting, etc. Due to these incomes there are different solutions. The first one is to overcome this area, but it is not always the most convenient - the alternative way is too far or too much time consuming. The next steps could be to try to overcome this area. If the snow is frozen, the area could be trafficable for example if you cover it with sand or gravel. The most complicated is the melting snow. It that case there is very high probability that the vehicles mire. Unfortunately, additional layer of gravel or sand does not help. The snow could be removing by vehicles, but this is very demanding to devices, equipment and time. So are there any means, which could be used to overcome the untrafficable terrain caused by the snow? The authors tried to examine the perspective materials - mats and stripes used for example for negotiation of low endurable terrain. Does this system work as well on snowy areas? Is it possible to use them? [4,5]

\section{Experimental Measurement}

\section{1. The Measurement Conditions}

The authors decided to verify the possibilities of using perspective materials by experimental testing is situ. It was chosen two types of perspective materials, three different areas covered by snow and three types of vehicles.

Place - The experimental measurement take place in a military area, 620 meters above sea level, the temperature was $5 \mathrm{oC}$ and there was bright and sunny weather. These conditions caused melting of the snow. There was chosen three places with different high of melting snow - from $15-40 \mathrm{~cm}$. Two places were on plateau and one was on slope. The place was marked by letters, A, B, C to be easier treated.

Vehicles - the representative personnel and truck vehicles of the Czech Army were chosen - UAZ 469 Bi, TATRA T-810 and Mitsubishi Pajero. Fig. 1.

UAZ $469 \mathrm{Bi}$ is an all-terrain vehicle manufactured by UAZ. The UAZ-469 presented two great advantages: It was able to drive in virtually any terrain and it was very easy to fix. The UAZ-469 reached legendary status for its reliability and off-road ability [9].

\footnotetext{
${ }^{1}$ Corresponding author.

E-mail address: klara.cibulova@unob.cz.
} 
Mitsubishi Pajero is personnel vehicle. It has earned a respected legacy in rally and are particularly noted for having won the Dakar Rally 12 times, having the highest number of Dakar Rally stage wins [9].

TATRA 810 is a medium class all-wheel-drive $(4 \times 4)$ off-road logistic truck that, unlike other TATRA trucks and chassis-cabs, is based on the standard chassis concept - rigid portal axles and a ladder frame. The truck was developed according to specifications given by the Czech Army for a replacement of their aging fleet of medium trucks. As it was required, this high mobility off-road truck has been designed at the borderline of medium and heavy truck classes [9].
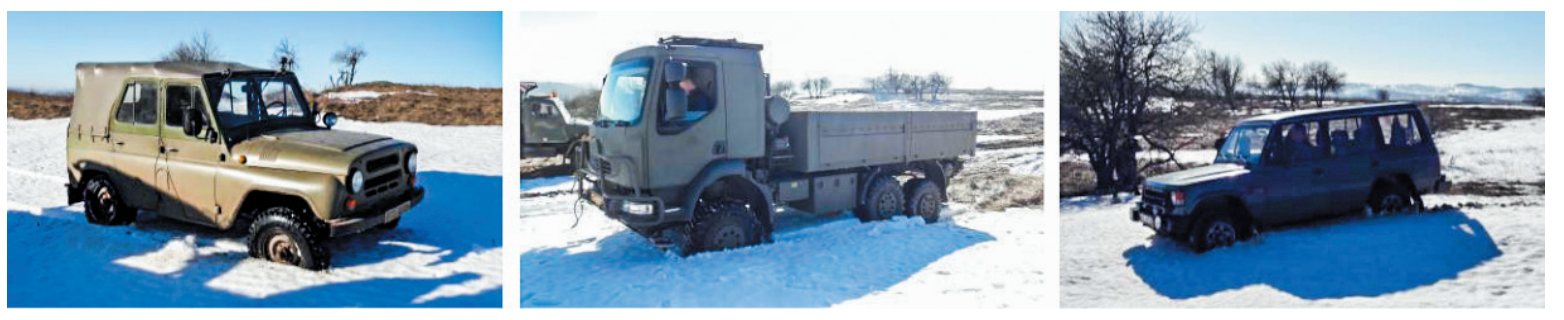

Fig. 1. The representative vehicles - UAZ, TATRA and Mitsubishi. They are shown in places, where they mired.

Perspective materials - these materials becoming more and more popular these days. There are different types - light or heavy ones. They are made in different widths and lengths. These materials have not been much known in the Czech Republic yet. That is why there are tested now different types in different conditions and with various types of vehicles. It is possible solution not only for the Army of the Czech Republic, but for all during the crisis situations.

Two types of perspective materials were chosen. The first one was the plastic mat (Light Trackway type) reinforced by metal rods. The length is $10 \mathrm{~m}$ and the width is $3 \mathrm{~m}$. The second were the Bogstripsset version $10 \mathrm{x} 0.5$ m. Fig. 3 Both are able to be operated only by two (four - depended on the length) people, so there is no need of any special equipment [10].

The plastic mats have to be placed and fixed very properly. They are provided with metal noose. There are two possible ways how to fix them. It could be fixed by special iron rod hammered into the ground or with a rope threated into the noose and fixed to a solid part, like tree etc. Also, the mats could be connected together. Fig. 5.

\section{2 Place A Testing}

The first place is A, it was on plateau, the high of snow layer was between $20-40 \mathrm{~cm}$. The tested vehicles were UAZ and TATRA. On this place the self-rescue and overcoming of snowy terrain with the help of perspective materials were tested. The measurement course was following.

The first vehicle UAZ go to the snowy area. After $5 \mathrm{~m}$ it mire. The driver firstly tried to self-rescue by using return, but was not successful. After that it was used the plastic strips (Fig. 2; Fig. 5). The vehicle was able to selfrescued and continued forward on the stripes. It mired again after few meters on terrain without the mats. After that the terrain was laid with the mats. It was used both, connected together. The vehicle UAZ was able to overcome this area without any problems. Also, after passing the mats, it mired after few meters.

Second tested vehicle was TATRA. The measurement course was similar. Firstly, the vehicle TATRA tried to overcome the area without any mats and it also mired. After that the stripes were used for self-rescuing of the vehicle. It was more demanding than with the personnel vehicle, but finally the attempt was successful. The last part of measurement on this place was overcoming this area with plastic mats. The truck easily passed the given area on mats, but after the mats ends the truck mired again. The overcoming distance are shown on Fig. 2.
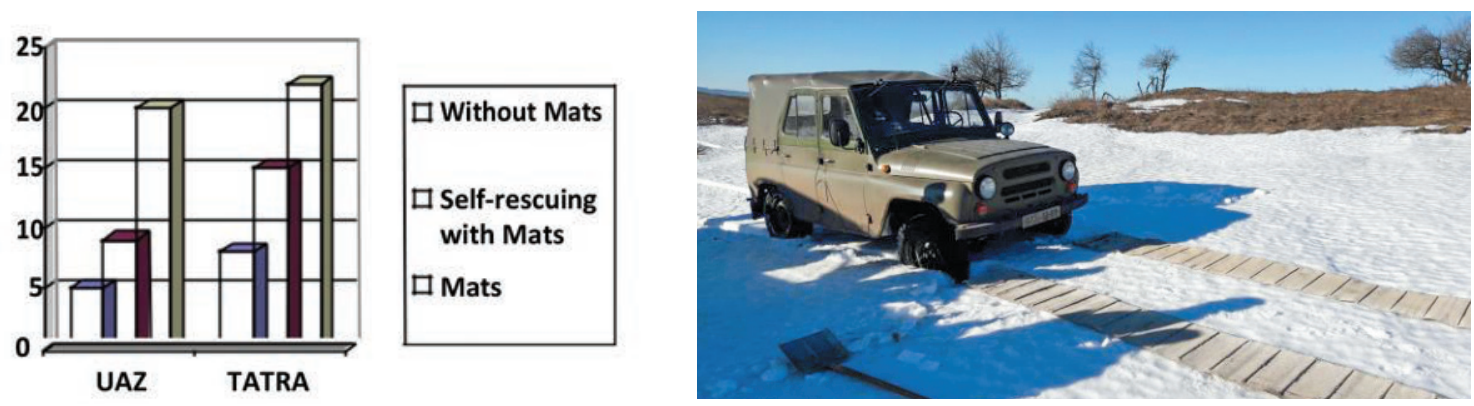

Fig. 2. The results of measuring on place A. A successful overcoming of UAZ with using the perspective materials. 


\section{3. Place B Testing}

The second place B was on slope, the high of snow layer was between 20 and $40 \mathrm{~cm}$. The tested vehicles were UAZ and TATRA. On this place it was testing the overcoming of snowy terrain with the help of perspective materials. The measurement course was following.

The first vehicle UAZ go to the snowy area. After $5 \mathrm{~m}$ it mire. Then the same area was laid with the mats. It was used both types, connected together. The vehicle UAZ was able to overcome this area without any problems. Also, after passing the mats, it mired after few meters.

Second tested vehicle was TATRA. The measurement course was similar. Firstly, the vehicle TATRA tried to overcome the area without any mats and it also mired. The truck easily passed the given area on mats, but after the mats ends the truck mired again. The overcoming distance are shown on Fig. 3.
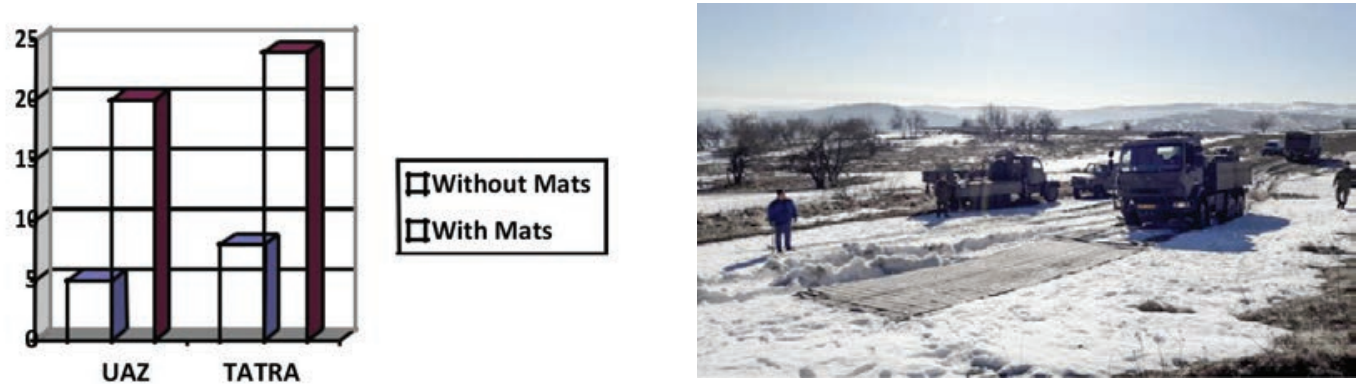

Fig. 3. The results of measuring on place B. A successful overcoming of TATRA with using the perspective materials.

\section{4. Place C Testing}

The third place $\mathrm{C}$ was on plateau. The high of snow layer was between 15 and $30 \mathrm{~cm}$. The tested vehicle was the Mitsubishi one. On this place it was testing the overcoming of snowy terrain with the help of perspective materials. The measurement course was following.

The personnel vehicle Mitsubishi tried to pass the snowy area. It mired after 4 meters. Then the same area was laid with the perspective material - connected stripes and mats. The vehicle was able to overcome this area without any problems. It was able to pass it to the area without snow and then went the same way back.
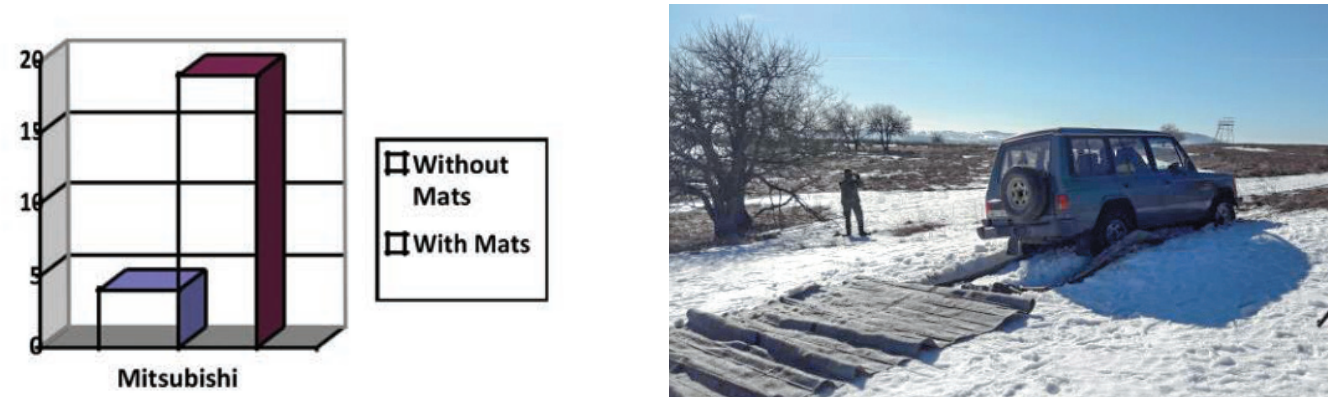

Fig. 4. The results of measuring on place C. A successful overcoming of Mitsubishi with using the perspective materials - both directions.

\section{5. Evaluations of the Measurement}

The aim of the experiment was to find out, if it is possible to use the perspective materials for the negotiation of the areas covered by snow and for the self-rescuing works. It was made seven experiments with different types of vehicles, materials and on three places. In two attempts the vehicles mired and they were able to self-rescued themselves by using the bogies. In the rest cases the vehicles mired, but after rescuing they were able to overcome the area with the mat or combination of mat and stripes. The investigation results are very affirmative, because it was proved that the perspective materials used for negotiation of low endurable terrain could be also used for negotiation of areas covered by snow. 

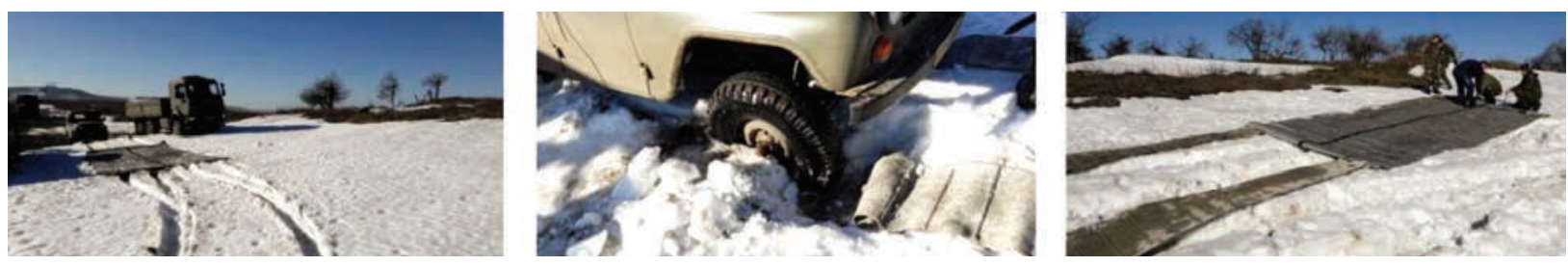

Fig. 5. The extension of a way with mats. The detail of self-rescuing of UAZ. Connection of two perspective materials.

\section{Conclusions}

The areas covered by snow represent considerable obstacle for the troops. The vehicles can mire and the mobility of the troop can be stopped. That is why the authors decided to verify, if it is possible to minimalize these obstacles and improve the mobility of the units. The following results of the investigation were obtained:

- The perspective materials can be used as self-rescuing means for vehicles mired in snow.

- The perspective materials can be used as the means for negotiation untrafficable areas covered by snow.

The results of this experiment verified that using of perspective material, such as plastic mats, could be the solution for negotiation areas covered by snow and for the self-rescuing of the vehicles. For this experiment there were chosen three vehicles, two types of plastic mats and three places. And the result of this experiment confirmed that the assumptions were right. The mobility of the troops would not be stopped and the means are very easy to use, in short time with few people. That is why the authors are planning to continue with these measurements and test more vehicles, more perspective materials and also different place with different slopes and conditions - like ice and others $[6,7]$.

The issue of military mobility has returned with vengeance. The mobility has to be ensured and the more areas will be trafficable for the troops the larger success could be expected. Each part of the mobility being dealt step by step is a little part of the puzzle which have to be solved to defeat the trafficability [8].

\section{Acknowledgements}

Presented work has been prepared with the support of the Ministry of Defence of the Czech Republic, Partial Project for Institutional Development, K-201, Department of Weapons and Ammunition, University of Defence.

\section{References}

1. Hoskova-Mayerova S, Hubacek M, Bekesiene S, Bures M. 2017. Vehicle movement modelling possibilities for defense and crisis management, Safety and Reliability - Theory and Applications - Epin \& Briš (Eds). London: Taylor \& Francis Group, 3035-3039.

2. Neumann, V., Krobot, Z. 2017. Analysis of track line parameters and their influence on track line stress. In: Transport Means 2017. Kaunas: Kaunas University of Technology, 2017, p. 788-793. ISSN 1822-296X.

3. Rybanský, M., Dohnal, F., Hosková-Mayerová, S., Svatonova, H. 2018. The impact of drainage on terrain UGV movement. In: 9th IGRSM International Conference and Exhibition on Geospatial \& Remote Sensing (IGRSM 2018) 24-25 April 2018, Kuala Lumpur, Malaysia. Kuala Lumpur, Malaysia: IOP Conference Series: Earth and Environmental Science, 169(1), 2018, ISSN 1755-1307.

4. Křístek, V., Kravtsov, A., Římal, J., Gattesco, N., Franceschinis, R. 2012. Strengthening Effectiveness of Ancient Masonry Bridges. 6th International Conference on Bridge Maintenance, Safety and Management. Cernobbio, Lake Como, Italy.

5. Bekesiene S. 2017. Mini Unmanned Aerial Vehicle System Optimization for Lithuanian Military Demands. Transport Means 2017. Kaunas: Kaunas University of Technology, 735-740. ISSN 1822-296X.

6. Rolenec, O., Kopuleny, M. 2017. Engineer Devices for Obstacle Breaching in Offensive Operations and Possible Application of Engineer Robots. In: INTERNATIONAL CONFERENCE ON MILITARY TECHNOLOGIES ICMT 2017. Piscataway, NJ 08854-4141 USA: Institute of Electrical and Electronics Engineers Inc., 2017, p. 200-206. ISBN 978-1-5386-1988-9.

7. Dohnal, F., Hubáček, M., Simková, K. 2019. Detection of Microrelief Objects to Impede the Movement of Vehicles in Terrain. ISPRS International Journal of Geo-Information, 2019, (3)(101), 1-16. ISSN 2220-9964.

8. Marko, M., Droppa, P., Lukášik, P., Marchevka, M. 2019. TATRA-815 engine oils degradation comparison of "Go-Stop" and Standard type of operation. Transport Means 2019. Palanga: Kaunas University of Technology. p 1553-1558. ISSN 1822-296X.

9. Vojenská zabazpečovací vozidla. Military supporting vehicles [online cit.: 2019-05-16]. Available from: http:// www.army.cz

10. R.V.M.B.V. Product Range [online cit.:2019-05-04]. Available from: hhtp://rvmbv.com 